\title{
Human Resource Development Review
}

\section{Learning to Do Qualitative Data Analysis: A Starting Point}

\begin{tabular}{|c|c|}
\hline Journal: & Human Resource Development Review \\
\hline Manuscript ID & HRDR-19-0144.R2 \\
\hline Manuscript Type: & Instructor's Corner \\
\hline Keywords: & Qualitative Research, qualitative data analysis, HRD \\
\hline Abstract: & $\begin{array}{l}\text { Given the vast and diverse qualitative analytic landscape, what might be } \\
\text { a generative starting point for researchers who desire to learn how to } \\
\text { produce quality qualitative analyses? This question is particularly } \\
\text { relevant to researchers new to the field and mentors who regularly } \\
\text { introduce students to qualitative research practices. In this article, we } \\
\text { seek to offer what we view as a useful starting point for learning how to } \\
\text { do qualitative analysis. We begin by discussing briefly the landscape of } \\
\text { qualitative research methodologies and methods. To contextualize our } \\
\text { suggestions, we review the qualitative analytic practices commonly used } \\
\text { in HRD. Following this, we describe thematic analysis in detail, including } \\
\text { why we believe it is a useful analytic approach to consider when first } \\
\text { learning about qualitative analysis. We share seven common practices or } \\
\text { considerations for carrying out a thematic analysis and conclude by } \\
\text { highlighting key considerations for assuring quality when conducting a } \\
\text { thematic analysis. }\end{array}$ \\
\hline
\end{tabular}

\section{SCHOLARONE Manuscripts}




\begin{abstract}
Given the vast and diverse qualitative analytic landscape, what might be a generative starting point for researchers who desire to learn how to produce quality qualitative analyses? This question is particularly relevant to researchers new to the field and practice of qualitative research and instructors and mentors who regularly introduce students to qualitative research practices. In this article, we seek to offer what we view as a useful starting point for learning how to do qualitative analysis. We begin by discussing briefly the general landscape of qualitative research methodologies and methods. To contextualize our suggestions, we review the qualitative analytic practices commonly used within HRD. Following this, we describe thematic analysis in more detail, including why we believe it is a particularly useful analytic approach to consider when first learning about qualitative analysis. We share seven common practices or important considerations for carrying out a thematic analysis and conclude by highlighting key considerations for assuring quality when conducting a thematic analysis.
\end{abstract}




\section{Learning To Do Qualitative Data Analysis: A Starting Point}

For many researchers unfamiliar with qualitative research, determining how to conduct qualitative analyses is often quite challenging. Part of this challenge is due to the seemingly limitless approaches that a qualitative researcher might leverage, as well as simply learning to think like a qualitative researcher when analyzing data. From framework analysis (Ritchie \& Spencer, 1994) to content analysis (Schreier, 2012) to discourse analysis (Wood \& Kroger, 2000), among many others, there are a plethora of distinct ways in which a researcher might complete their qualitative analysis. Even what many argue to be a mainstay qualitative analytic approach, that is, thematic analysis, has been conceptualized in varying ways and is arguably better understood as an umbrella term that captures within it a set of very different approaches to analysis (see Braun, Clarke, Hayfield \& Terry, 2019, for a discussion of the history of thematic analysis). Given this vast and diverse qualitative analytic landscape, what might be a generative starting point for researchers who desire to learn how to produce quality and useful qualitative analyses? This question, we suggest, is particularly relevant to researchers new to the field of qualitative research - our primary audience - as well as instructors and mentors who regularly introduce students to qualitative research practices.

In this paper, we take up this open question as a point of departure and offer thematic analysis, an analytic method commonly used to identify patterns across language-based data (Braun \& Clarke, 2006), as a useful starting point for learning about the qualitative analysis process. In doing so, we do not advocate for only learning the nuances of thematic analysis, but rather see it as a useful beginning point. Arguably, it is through exposure to diverse modes of analysis that researchers begin "to imagine new possible configurations for research" (Freeman, 2017, p. 4). Thus, what we offer in this paper is an abbreviated roadmap for human resource 
development (HRD) scholars and students who may be unfamiliar with qualitative analysis. Further, in this paper, we do not aim to offer a new approach to qualitative analysis; rather, we share a roadmap that we - along with many other scholars (see Clarke \& Braun, 2013, for an example, among many others) - have found useful when introducing people to the practice of doing qualitative analysis.

To begin, we discuss the general landscape of qualitative research methodologies and methods. Specifically, we briefly review the current state of qualitative analysis in HRD. Following this, we describe thematic analysis in more detail, including why we believe it is a particularly useful (but not the only) approach to consider, and outline seven practices or important considerations for carrying out a thematic analysis. We conclude by highlighting key considerations for assuring quality when conducting a thematic analysis.

\section{The Landscape of Qualitative Approaches to Analysis}

Broadly, qualitative research is generally employed to support a researcher in generating a deep and nuanced understanding of a given phenomenon. The outcomes of such research range from generating findings that can inform practice (e.g., Lochmiller, 2016) to providing detailed descriptions of a given problem of practice (e.g., Honig, 2006) to offering insights about professional practices within a given context (e.g., O’Reilly et al., 2015) and tackling issues related to the subjective nature of qualitative research (e.g., Cho et al., 2016), among others. The potentiality of conducting qualitative research is notable and yet dependent upon researchers being able to conduct grounded, rigorous analyses, and more generally, understanding what it means to do qualitative analysis.

Broadly conceived, qualitative data analyses bring meaning to a dataset (Anfara, Brown, \& Mangione, 2002), with qualitative data including a wide range of materials (e.g., 
conversational data, images, observations, and unstructured, semi-structured, or structured interviews, among others). Indeed, qualitative data analysis means various things, as it is often aligned with a particular methodology, theoretical perspective, research tradition, and/or field (Lochmiller \& Lester, 2017). Coffey and Atkinson (1996) remind us that, "there is no single right way to analyze qualitative data; equally, it is essential to find ways of using the data to think with" (p. 2). Miles and Huberman (1994) argued that there are several common practices that often (but not always) persist across qualitative approaches to analysis, including:

- Affixing codes to a set of field notes drawn from observations or interviews

- Noting reflections or other remarks in the margins

- Sorting and sifting through these materials to identify similar phrases, relationships between variables, patterns, themes, distinct differences between subgroups, and common sequences

- Isolating these patterns and processes, commonalities and differences, and taking them out to the field in the next wave of data collection

- Gradually elaborating a small set of generalizations that cover the consistencies discerned in the data

- Confronting those generalizations with a formalized body of knowledge in the form of constructs or theories. (p. 9)

While there are certainly common analytic practices, there are also a range of ways that one might analyze qualitative data, with each approach bringing unique theoretical assumptions and expectations. Given that it is beyond the scope of this paper to overview the many different ways one might analyze qualitative data (see Freeman, 2017, for a thorough discussion of analytic perspectives in qualitative research), we instead provide an abbreviated snapshot of the 
approaches that some scholars within HRD are engaging. Our aim in doing so is to contextualize our discussion in relation to HRD in particular.

Thus, to capture a snapshot of the current state of qualitative research and qualitative data analysis practices in HRD, we conducted a review of Human Resource Development Quarterly $(H R D Q)$ between 1990 and the current issue (Vol. 33, No. 3) in 2019 and identified 59 qualitative articles. The types of qualitative research included: 24 case studies, 19 generic qualitative studies, and eight phenomenological studies. Notably, about half of the articles reported analyzing their qualitative data via content analysis and a constant comparative method, which was also commonly referred to as a grounded theory approach and/or inductive analysis. Four studies did not identify data analysis methods, and no mention was made of using a qualitative data analysis software package, such as NVivo or ATLAS.ti, until articles published in 2007 (see Table 1).

$<<<$ Insert Table 1 about here $>>>$

Across the articles identified, a grounded theory approach to analysis appeared to be the go-to qualitative analysis method, with in-depth and semi-structured interviews being the most common data types. It was not uncommon for some of the authors to attribute analytic activities linked to grounded theory and the associated constant comparative method to thematic analysis, as evidenced in the literature we reviewed. Relatedly, some scholars have argued for the importance of recognizing that thematic analysis is not uniformly enacted (Braun \& Clarke, 2016), as there is notable diversity in how it is conceptualized and carried out (e.g., Braun \& Clarke, 2003; Boyatzis, 1998).

\section{Thematic Analysis}


Thematic analysis is perhaps best defined as "an umbrella term, designating sometimes quite different approaches aimed at identifying patterns (themes) across qualitative datasets" (Braun et al., 2019, p. 844). Due to its broad and flexible nature, thematic analysis has been widely used in diverse fields including psychology (Frith \& Gleeson, 2004), medicine (Cassol et al., 2018), health services (Norris et al., 2017), tourism (Costa et al., 2016), HRD (Israel et al., 2017; Perkins, 2018; Tsai, 2016), and education (Halverson et al., 2014).

\section{Why Thematic Analysis?}

Importantly, in forwarding thematic analysis, we are not suggesting that it is useful for all qualitative research designs or even that it is the most innovative approach. In fact, we caution against viewing thematic analysis as the end all, be all approach for analyzing qualitative data, agreeing with Wang and Roulston's (2007) assessment that "the diverse field of qualitative inquiry offers many other data analysis techniques that have yet to be taken up by qualitative inquirers in HRD” (p. 181). Instead, we argue that learning how to conduct a thematic analysis serves as an important foundation for eventually making sense of other, more specialized forms of analysis. Further, like Clarke and Braun (2013), in our own teaching of qualitative methods, we have found thematic analysis to be "a useful - and a relatively easy to teach and learn - basic introduction to qualitative analysis" (p. 120).

We thus view thematic analysis as a useful starting point for learning and doing rigorous qualitative analyses for several reasons. First, we view thematic analysis as offering tremendous "theoretical flexibility" and potentially being used as "just an analytic method, rather than a methodology, which most other qualitative approaches are" (Clarke \& Bruan, 2013, p. 120). This theoretical flexibility allows researchers across a range of disciplines to engage disciplinary theories and perspectives when conducting a thematic analysis, potentially generating a more 
meaningful and relevant analysis for a given field. Accordingly, thematic analysis can result in a theory-driven or data-driven set of findings and engage a range of research questions (Braun $\&$ Clarke, 2006). Second, thematic analysis engages with analytic practices that are fairly common with other approaches to qualitative analysis. For instance, when conducting a thematic analysis, it is common to sort and sift through the dataset to identify similar phrases and/or relationships; a practice that has been described as being common across many qualitative analytic approaches (Miles \& Huberman, 1994). Third, thematic analysis can be used when analyzing various kinds of data, as well as a range of dataset sizes.

\section{Conducting a Thematic Analysis}

Qualitative data analysis is generally described as a non-linear, iterative process. Thus, it is common for researchers not to list out a step-wise analytic process. We suggest, however, that it is quite useful to think about the process in relation to phases - which are certainly often overlapping. These phases can be pursued in a systematic way, while also recognizing that qualitative analysis is fundamentally flexible and even a bit messy. The value of structuring data analysis in phases is that it creates a transparent process for both the qualitative researcher and (ultimately) the reader of a given research report. Borrowing from Lochmiller and Lester's (2017) earlier work, we offer here seven phases to engage when completing a qualitative analysis. These phases, we suggest, are particularly well-suited for thematic analysis, wherein the researcher aims to produce broad descriptive statements that reflect their overall understanding of the data and in response to their research questions. These phases include: preparing and organizing the data, transcribing the data, becoming familiar with the data corpus, memoing the data, coding the data, producing categories and themes from underlying coded passages, and making the analysis process transparent. We describe these phases below. 
Phase One: Preparing and organizing the data for analysis. Qualitative fieldwork often generates a considerable data corpus. Hours of interviews or focus groups, pages of observational notes, and countless documents can be retrieved during the data collection. The amount of data collected is often conditioned on the length of the fieldwork phase. Thus, one of the first steps in qualitative analysis is to prepare and organize the data for thematic analysis. In practice, this typically involves gathering all of the audio- or video-recorded interview files into one location, converting observational notes to electronic format (e.g., MS Word documents or Adobe PDF), and scanning documents retrieved in paper form. We recommend that this process include a structured naming protocol for each file, as well as the production of a master data catalogue that lists each data source, its storage location, its creator, and the date of its collection. Although simplistic, this stage is important as it is at this point that scholars begin developing the data corpus from which they will complete their thematic analysis. Furthermore, it serves as an opportunity to set up the dataset so that it can be imported into a qualitative data analysis software package, such as ATLAS.ti, MAXQDA, or NVivo.

Phase Two: Transcribing the data. Given that audio or video data is commonly collected in qualitative research, qualitative researchers generally allocate time to transcribing the data in preparation for further analysis. There are several different kinds of transcripts used in qualitative research (e.g., multimodal transcript, gisted transcript, verbatim transcript), which links closely to the methodology used and purpose of a given study. For thematic analysis, verbatim transcripts are quite common; that is, transcripts that aim to capture every utterance from the participant and serve as an accurate record of the conversation.

Transcribing a dataset can feel overwhelming and it may be tempting (and at times necessary) to outsource this activity to a professional transcriptionist. Yet, transcription serves as 
an opportunity to become familiar with a dataset. This familiarity deepens a researcher's understanding of the participants' perspectives and supports them in understanding the dataset in a way that accelerates analysis later on. Thus, we strongly encourage scholars to generate their own transcripts as opposed to hiring a professional transcriptionist to transcribe the audio or video files. At the same time, we recognize that there are a growing number of automated transcription options (e.g., Temi, Trint, etc.) that are changing the place and practice of transcription, as well as possibilities for directly coding audio and video files within QDAS packages (Paulus, Lester, \& Dempster, 2014). Importantly, then, we encourage qualitative researchers to consider technological innovations related to transcription when determining how and to what degree they transcribe their dataset.

Phase Three: Becoming familiar with the data. Once organized and transcribed, researchers must become familiar with the data they collected. It can be helpful to think of this step as light or initial analysis, wherein scholars take note of the ideas or experiences described by participants that appear in interviews, recorded in observation notes, or described in documents. These initial understandings can often inform a researcher's later, more detailed analysis. Thus, it is important to be aware of and chronicle in detail these early reactions to the data. It also helps to become familiar with the corpus of data so that a researcher is aware of the limitations or gaps in the collected data. This might inspire further data collection or marking of these gaps as areas for further research.

Phase Four: Memoing the data. As researchers review their data, it can be helpful to generate memos that describe initial reflections about the data, as well as any emergent interpretations. These memos are, in simple terms, a "conversation with ourselves about our data" (Clarke, 2005, p. 202). They are designed to capture emergent understandings, as well as to 
denote potential biases that may influence the interpretation of the data. Memos can also capture places in the data where there is - or will be - statements or experiences that are potentially of analytic importance. For instance, an HRD professional's reflection on a new professional development program might be of analytic importance in a study of the effectiveness or impact of the program. A researcher may wish to construct a memo which documents their early interpretation of the significance of this reflection, as well as its connection to other aspects of the study (e.g., theoretical framework). Importantly, memos “should be suggestive; they needn't be conclusive" (Dey, 1993, p. 89). Thus, memos serve as an invitation for further analysis. Given the advent of qualitative data analysis software packages, these memos can be generated electronically and be directly linked to specific segments of the data; that is, researchers can attach memos directly to primary data sources. This allows a researcher to retrieve both their written memo and the segment of data that prompted its development.

Phase Five: Coding the data. A particularly important part of the thematic analysis process involves coding the data. A code is simply a short, descriptive word or phrase that assigns meaning to the data related to the researcher's analytic interests. Although coding is often completed in an unstructured manner, we argue that for a thematic analysis it is important to think of coding as occurring in multiple phases (see Saldana, 2016, for further discussion of coding in cycles).

While we do not advocate for a particular number of coding phases or cycles, we suggest thinking about at least three phases. In the first phase, a researcher generally assigns codes to the entire dataset. This layer of coding serves to identify important statements, experiences, and reflections. As such, the first layer of coding is primarily about priming the dataset and seeking to reduce the size of the data corpus by denoting those statements, experiences, and reflections 
that are of analytic importance. The codes assigned in the first phase are often descriptive in nature and reflect a relatively low level of inference. In the second phase, researchers frequently return to the passages/data segments they assigned codes to in the first phase and assign additional codes. Codes at this stage begin moving to a higher level of inference, particularly as a researcher begins reflecting concepts and/or ideas that are more directly related to the focus of the study. The aim of the second phase of coding is to begin connecting statements, experiences, and reflections offered by research participants to the study's analytic interests. Finally, researchers generally complete a third phase of coding wherein they make explicit connections to the study's conceptual and/or theoretical ideas. In this phase, coding typically reaches its highest level of inference. While earlier phases of coding sought to identify what was happening in the dataset for the purposes of reducing the dataset in size and complexity, codes at this phase connect these statements, experiences, and reflections with specific conceptual or theoretical ideas. For example, a statement or comment coded in prior rounds of coding may be specifically highlighted as an example of a conceptual or theoretical idea.

Phase Six: Moving from codes to categories and categories to themes. Broadly, thematic analysis involves inductive engagement with the data, with researchers moving from isolated cases to broader interpretations. This process involves the application of codes, development of categories, and ultimately the production of themes. Patton (1980) noted that "inductive analysis means that the patterns, themes, and categories of analysis come from the data; they emerge out of the data rather than being imposed on them prior to data collection and analysis" (p. 306). Thus, codes represent the earliest stage in the analytic process.

Metaphorically, codes can be thought of as individual puzzle pieces which, as a collection, contribute to a researcher's depiction of the data. Individually, codes do not tell the entire story. 
Rather, in order for that to occur, the researcher must understand how codes (inter)relate and contrast with one another. These (inter)relationships and contrasts are encapsulated in the categories. Categories aggregate individual codes that are related analytically or conceptually. This aggregation represents an important intermediate step in the production of themes.

Once categories have been developed, then researchers aim to produce their themes. This involves two steps. First, a researcher must bring together various related categories. This involves recognizing the similarities, differences, and relationships across categories. Second, with these similarities, differences, and relationships acknowledged, the researcher then assigns a statement to these categories. This theme name should be inclusive of all of the underlying categories, as well as descriptive of their content, the relationships between them, as well as being responsive to any similarities or differences observed. Themes are generally aligned with the conceptual or analytic goals of the study and therefore are designed in response to the study's primary research questions or focus.

Phase Seven: Making the analytic process transparent. An important consideration when conducting a thematic analysis is to present information about the analytic process in a transparent and verifiable manner. There are indeed a range of ways that researchers might pursue this, and we only offer three possibilities here.

First, we have found creating a map of the analytic process to be a particularly useful way by which to share one's process of moving from codes to categories to themes. Mapping the process supports a researcher in being open about the development of themes and allows for outside readers/evaluators of a given research study to discern how a researcher went about making key analytic choices (Anfara et al., 2002). 
Second, to support transparency, we recommend that researchers develop a detailed audit trail that delineates the connection between data sources, codes, categories, and themes. Many researchers have taken to presenting this audit trail in tabular form and including the information in the published manuscript. This approach involves selecting a few representative segments from a dataset, indicating which codes were initially applied to this segment, and then articulating which categories and themes the segment is responsive to. This approach ensures that the researcher's interpretation and coding process is visible to an outside reader/evaluator and thus builds trustworthiness in relation to the interpretations of the dataset.

Finally, another strategy involves reporting coding usage in frequencies. While reporting frequencies may not be preferred by all researchers, in some disciplines and venues, it is valued and even preferred. This process can be greatly assisted by the use of qualitative data analysis software packages. These packages not only tabulate coding frequencies but also allow these tabulations to be exported in publication-ready formats. Coding frequencies illustrate how densely codes were applied to the dataset and thus enable readers to understand the extent to which codes were used to derive the qualitative themes. More importantly, reporting coding frequencies can provide some indication about how representative a theme may be in relation to the larger dataset.

\section{Completing a Quality Analysis}

Phase Seven above addresses, at least in part, concerns related to quality. Nonetheless, given that this is a particularly salient consideration for researchers across fields, we offer here a few additional considerations for pursuing and completing a qualitative analysis.

The diversity and heterogeneity of qualitative research is reflected in the varying arguments that exist about how to establish quality and claim rigor. The literature has 
emphasized the importance of ensuring rigor and transparency in qualitative research, calling for a revisit of the evaluation criteria on qualitative research, particularly concerning qualitative data analysis. For instance, a recent analysis of 52 qualitative articles in the Strategic Management Journal revealed that none of the published studies were sufficiently transparent, with insufficient transparency being potentially pervasive in qualitative research (Aguinis \& Solario, 2019; Anfara et al., 2002). Further, Anderson (2017), in her article focused on evaluation criteria for qualitative research, argued that qualitative researchers must pursue rigor in qualitative research (at least) to the same degree as quantitative researchers do. To that end, she recommended 10 evaluation criteria for rigor in qualitative research, and four of them are related to qualitative data analysis: communication of methodological awareness (e.g., audit trail), member-checking procedures, triangulation, and peer debriefing. Similarly, Rocco’s (2010) criteria for evaluating qualitative studies included a data analysis process described in detail with limitations reported. On the basis of Rocco (2010), Storberg-Walker's (2012) amended list on qualitative data analysis in research papers included the following: (a) the article should provide enough details so that reviewers could follow the same analytical steps; (b) the analysis process selected should be logically connected to the purpose of the study; and (c) the analysis process should be justified.

Indeed, there have long been discussions of how to establish criteria. Lincoln and Guba (1985) offered the classic articulation of four criteria (i.e., credibility, transferability, dependability, and confirmability) and related techniques (e.g., prolonged engagement in the field, thick descriptions, and audit trails) for establishing criteria to Tracy's (2010) more recent presentation of eight markers of quality. Yet there remains very little consensus around what counts as quality in qualitative research, with a range of positions taken - often related to unique 
theoretical perspectives, methodological preferences, and field-specific norms. When examining the qualitative methodological literature, O’Reilly and Kiyimba (2015) identified at least four ways in which quality (sometimes referred to as validity, among other terms) has been characterized. The first position on quality utilizes quantitative conceptualizations and simply modifies and adapts the core notions of validity, reliability, and generalizability. The second position relates to the development of qualitative-specific universal markers of quality (e.g., Tracey, 2010), which draws upon qualitative vocabulary (e.g., transferability rather than generalizability). The third position casts quality in terms of specific methodological boundaries, arguing that universal criteria are not particularly useful and thus unique (albeit often overlapping) quality criteria are needed which align with the assumptions of a given qualitative methodology or analytic approach (e.g., thematic analysis). The fourth position denies the need for quality indicators.

We suggest when conducting thematic analysis that a useful starting point for establishing quality is both taking into account discussions about universal criteria (e.g., Tracy, 2010), as well as practices specific to thematic analysis (e.g., Terry, Hayfield, Clarke \& Braun, 2017). For instance, in Terry et al.'s (2017) discussion of quality related to thematic analysis - as conceptualized by Braun and Clarke (2006) - they noted several common inconsistencies that impact quality. These inconsistencies range from incongruous use of a theoretical perspective when interpreting data to combining theoretically and methodologically distinct approaches to thematic analysis to generating themes that are nothing more than the very questions posed to participants, among others. A particularly useful starting point for assessing the quality of a given thematic analysis is to turn to Braun and Clarke's (2006) 15-point checklist for conducting a qualitative thematic analysis. Importantly, this checklist is specifically related to the approach 
to thematic analysis articulated by Braun and Clarke (2006) and thus nuances across the various approaches to thematic analysis should always be closely considered.

\section{Conclusion}

In this paper, we have offered a roadmap for introducing individuals who are new to qualitative research to the process of conducting analysis, specifically a thematic analysis. We have argued that thematic analysis is a useful starting point for learning how to conduct qualitative analysis, as it provides a foundation for learning other approaches. Notably, what we offer here is only one of many ways to think about completing a thematic analysis. Thus, we encourage readers to engage with the methodological and disciplinary literature (e.g., Freeman, 2017; Saldana, 2916) to acquire a more nuanced understanding of the range of ways in which thematic analysis might be completed. 
LEARNING TO DO QUALITATIVE DATA ANALYSIS

\section{References}

Aguinis H., \& Solarino, A. M. (2019). Transparency and replicability in qualitative research: The case of interviews with elite informants. Strategic Management Journal. https:// doi.org/10.1002/smj.3015

Anderson, V. (2017). Criteria for evaluating qualitative research. Human Resource Development Quarterly, 28(2), 125-133. https://doi.org/10.1002/hrdq.21282

Anfara Jr, V. A., Brown, K. M., \& Mangione, T. L. (2002). Qualitative analysis on stage: Making the research process more public. Educational Researcher, 31(7), 28-38.

Boyatzis, R. E. (1998). Transforming qualitative information: Thematic analysis and code development. Thousand Oaks, CA: SAGE.

Braun, V., \& Clarke, V. (2006). Using thematic analysis in psychology. Qualitative Research in Psychology, 3, 77-101. doi: 10.1191/1478088706qp063oa

Braun, V., \& Clarke, V. (2016). (Mis)conceptualising themes, thematic analysis, and other problems with Fugard and Potts' (2015) sample-size tool for thematic analysis. International Journal of Social Research Methodology, 19(6), 739-743. https://doi.org/10.1080/13645579.2016.1195588

Braun, V., Clarke, V., Hayfield, N., \& Terry, G. (2019). Thematic analysis. In P. Liamputtong (Ed.), Handbook of research methods in health social sciences (pp. 843-860). Thousand Oaks, CA: SAGE.

Cassol, H., Pétré, B., Degrange, S., Martial, C., Charland-Verville, V., Lallier, F., Bragard, I., Guillaume, M., \& Laureys, S. (2018). Qualitative thematic analysis of the phenomenology of near-death experiences. PLoS ONE, 13(2), e0193001. https://doi.org/10.1371/journal.pone.0193001 
Cho, Y., Park, J., Ju, B., Han, S., Moon, H., Park, S., Ju, A., \& Park, E. (2016). Women leaders' work-life imbalance in South Korean companies: A collaborative qualitative study. Human Resource Development Quarterly, 27(4), 461-487. https://doi.org/10.1002/hrdq.21262

Clarke, A. E. (2005). Situational analysis: Grounded theory after the postmodern turn. Thousand Oaks, CA: SAGE.

Clarke, V., \& Braum, V. (2013). Teaching thematic analysis: Overcoming challenges and developing strategies for effective learning. The Psychologist, 26(2), 120-123.

Coffey, A., \& Atkinson, P. (1996). Making sense of qualitative data: complementary research strategies. Thousand Oaks, CA: SAGE.

Costa, C., Breda, Z., Pinho, I., Bakas, F., \& Durão, M. (2016). Performing a thematic analysis: An exploratory study about managers' perceptions on gender equality. The Qualitative Report, 21(13), 34-47.

Dey, I. (1993). Qualitative data analysis: A user-friendly guide for social scientists. London: Routledge.

Freeman, M. (2017). Modes of thinking for qualitative data analysis. London: Routledge.

Frith, H., \& Gleeson, K. (2004). Clothing and embodiment: Men managing body image and appearance. Psychology of Men \& Masculinity, 5(1), 40-48.

Halverson, L. R., Graham, C. R., Spring, K. J., Drysdale, J. S., \& Henrie, C. R. (2014). A thematic analysis of the most highly cited scholarship in the first decade of blended learning research. Internet and Higher Education, 20, 20-34.

https://doi.org/10.1016/j.iheduc.2013.09.004 
Honig, M. I. (2006). Street-level bureaucracy revisited: Frontline district central-office administrators as boundary spanners in education policy implementation. Educational Evaluation and Policy Analysis, 28(4), 357-383.

https://doi.org/10.3102/01623737028004357

Israel, T., Bettergarcia, J. N., Delucio, K., Avellar, T. R., Harkness, A., \& Goodman, J. A. (2017). Reactions of law enforcement to LGBTQ diversity training. Human Resource Development Quarterly, 28(2), 197-226. https://doi.org/10.1002/hrdq.21281

Lincoln, Y., \& Guba, E. (1985). Naturalistic inquiry. Newbury Park, CA: Sage.

Lochmiller, C. R. (2016). Examining administrators' instructional feedback to high school math and science teachers. Educational Administration Quarterly, 52(1), 75-109. https://doi.org/10.1177/0013161X15616660

Lochmiller, C. R., \& Lester, J. N. (2017). An introduction to educational research: Connecting methods to practice. Thousand Oaks, CA: SAGE.

Miles, M. B., \& Huberman, A. M. (1994). An expanded sourcebook: Qualitative data analysis ( $2^{\text {nd }}$ ed.). Thousand Oaks, CA: SAGE.

Norris, J. M., White, D. E., Nowell, L., Mrklas, K., \& Stelfox, H. T. (2017). How do stakeholders from multiple hierarchical levels of a large provincial health system define engagement? A qualitative study. Implementation Science, 12.

doi: 10.1186/s13012-017-0625-5

O'Reilly, M., \& Kiyimba, N. (2015). Advanced qualitative research: A guide to using theory. Thousand Oaks, CA: SAGE. 
O'Reilly, M., Karim, K., Stafford, V., \& Hutchby, I. (2015). Identifying the interactional processes in the first assessments in child mental health. Child and Adolescent Mental Health, 20(4), 195-201. https://doi.org/10.1111/camh.12077

Patton, M. Q. (1980). Qualitative evaluation methods. Thousand Oaks, CA: SAGE.

Paulus, T., Lester, J. N., \& Dempster, P. (2014). Digital tools for qualitative research. London, UK: SAGE.

Perkins, G. (2018). How does self-direction within learning operate to affect idea generation in small-medium enterprise contexts? Human Resource Development Quarterly, 29, 307328. https://doi.org/10.1002/hrdq. 21326

Ritchie, J., \& Spencer, L. (1994). Qualitative data analysis for applied policy research. In A. Bryman \& B. Burgess (Eds.), Analyzing qualitative data. Available from: http://dx.doi.org/10.4324/9780203413081_chapter_9

Rocco, T. S. (2010). Criteria for evaluating qualitative studies. Human Resource Development International, 13(4), 375-378.

Saldana, J. (2016). The coding manual for qualitative researchers ( $3^{\text {rd }}$ ed.). Thousand Oaks, CA: SAGE.

Schreier, M. (2012). Qualitative content analysis in practice. Thousand Oaks, CA: SAGE.

Storberg-Walker, J. (2012). Tips for publishing and reviewing qualitative studies in applied discipline. Human Resource Development Review, 11(2), 254-261. doi: $10.1177 / 1534484312436709$

Terry, G., Hayfield, N., Clarke, V., \& Braun, V. (2017). Thematic analysis. In C. Willing \& W. S. Rogers (Eds.), The SAGE Handbook of Qualitative Research in Psychology (2 $2^{\text {nd }}$ ed.) (pp. 17-37). Thousand, Oaks, CA: SAGE. 
Tracy, S. J. (2010). Qualitative quality: Eight "big tent" criteria for excellent qualitative research. Qualitative Inquiry, 16(1), 837-851. https://doi.org/10.1177/1077800410383121

Tsai, C.-J. (2016). Boredom at work and job monotony: An exploratory case study within the catering sector. Human Resource Development Quarterly, 27(2), 207-236. https://doi.org/10.1002/hrdq.21249

Wang, J., \& Roulston, K. J. (2007). An alternative approach to conceptualizing interviews in HRD research. Human Resource Development Quarterly, 18(2), 179-210. https://doi.org/10.1002/hrdq.1199

Wood, L. A., \& Kroger, R. O. (2000). Doing discourse analysis: Methods for studying action in talk and text. Thousand Oaks, CA: SAGE. 
Table 1

Qualitative Data Analysis Methods Used in 59 Qualitative Studies in HRDQ

\begin{tabular}{l|c}
\hline \multicolumn{1}{c|}{ Qualitative Data Analysis Method } & Number (\%) \\
\hline Constant Comparative Analysis/Grounded Theory Approach/Inductive Analysis & $20(33.9)$ \\
\hline Content Analysis & $12(20.3)$ \\
\hline Thematic Analysis & $3(5.1)$ \\
\hline Basic Interpretive Analysis & $2(3.4)$ \\
\hline Discourse Analysis & $1(1.7)$ \\
\hline Not Available & $4(6.8)$ \\
\hline Other (e.g., Template Analysis, Frame Analysis) & $17(28.8)$ \\
\hline
\end{tabular}

